\title{
MULTI-COINCIDENCE STUDIES OF FRAGMENTATIONS OF CORE EXCITED OCS MOLECULES
}

\author{
P. Erman, A. KarawajczyK, E. Rachlew, M. Stankiewicz* \\ AND K. YOSHIKI-Franzen
}

Department of Physics I, Royal Institute of Technology, 10044 Stockholm, Sweden

Molecular fragmentation following photon excitation of core electrons from $K$ shells of $\mathrm{C}$ and $\mathrm{O}$ and $L$ shell of $\mathrm{S}$ atoms forming the OCS molecule was studied. Emerging photoelectrons and charged fragments were detected in coincidence. Application of time-of-flight spectroscopy together with triple coincidence photoelectron-photoion-photoion, double coincidence photoelectron-photoion and photoion-photoion techniques allow a detailed insight into the geometry and the dynamics of the de-excitation processes and an unambiguous assessment of all fragmentation channels of highly excited molecules. Such studies are of major interest in fundamental research and they are relevant also in environmental, biological and technological investigations.

PACS numbers: 33.80.Eh, 33.80.Gj

\section{Introduction}

The dynamics of core-excited molecules are of interest from the point of view of fundamental physics - in the assignment of energetic structure of excited molecular ions or in angular distributions of the emerging fragments [1] for example, as well as of possible practical applications, for example the control of chemical reactions.

The processes following the photon excitation of a core electron of one of the molecular constituents can undergo many different scenaria leading to the production of the charged fragments. Generally, they can be divided into direct, one-step processes, and indirect ones like autoionization or predissociation which in turn can take multi-step paths. Usually, a number of decay channels are available simultaneously, with non-resonant processes of direct valence ionization contributing to the production of the fragments. Therefore unambiguous assignment of the decay channels of the core excited states and their branching ratios is difficult.

*Permanent address: Institute of Physics, Jagiellonian University, Reymonta 4, 30-059 Kraków, Poland. 
We present here results of multi-coincidence experiments, e.g. detection of the emerging electrons in coincidence with the charged molecular fragments and measurement of their kinetic energy released during the break-up, which gives us the required information [2-4].

\section{Experiment}

Our experiment was performed in MAX Laboratory - the Swedish synchrotron radiation source - at undulator beamline 51 . Soft $\mathrm{X}$-rays produced by the undulator in the energy range $100-600 \mathrm{eV}$ were energy selected by the modified SX-700 type monochromator. The coincidence apparatus comprises a Wiley-McLaren [5] type of ion time-of-flight (TOF) analyzer and a non-selective electron detector. The TOF analyzer has $210 \mathrm{~mm}$ long free drift region and the extracting and accelerating electric fields were adjusted to 400 and $1776 \mathrm{~V} / \mathrm{cm}$ respectively in order to fulfill the spatial focusing condition. The photon beam excited investigated molecules confined in a simple effusive jet produced at the output of a syringe needle. The background pressure in the experimental chamber was kept at $10^{-5}$ torr level typically. The raw signals from the detectors (25 $\mathrm{mm}$ diameter micro-channel plates) were amplified by fast preamplifiers EG\&G ORTEC VT120 and via fast discriminators TENNELEC TC 455 fed to start and stop inputs of a high resolution (500 ps) multi-channel scaler (MCS) FAST 7886 IBM PC card.

\section{Results}

All measurements presented below were performed at $288 \mathrm{eV}$ photon energy, corresponding to resonant $\pi^{*}$ excitation of $K$ shell electron of $\mathrm{C}$ atom of OCS molecule. The length of the TOF analyzer drift tube, the time resolution of the MCS card, the size of the photon beam $\left(\approx 10^{-5} \mathrm{~m}\right)$ and the spatial focusing condition fulfilled provide a high accuracy in the measurements of the kinetic energies of the fragments.

\subsection{The PEPICO experiment}

Figure 1 shows the photoelectron-photoion coincidence (PEPICO) spectrum. A number of emerging charged fragments are visible with the widths of the corresponding peaks being proportional to their initial momenta. Here, only electron-ion correlations are registered providing limited information e.g. the survey spectrum of all emerging charged fragments or the partial cross-sections for their production. The width of the parent dication peak gives us the valuable information on the thermal energy and the instrumental width. The measured width of $\mathrm{OCS}^{2+}$ peak in Fig. 1 is 4 ns which corresponds to the thermal energy being less than $20 \mathrm{meV}$ since no instrumental width was taken into account. Thus, the temperature of the effusive jet can be estimated to be below $300 \mathrm{~K}$.

\subsection{The PIPICO experiment}

The photoion-photoion coincidence (PIPICO) spectrum in Fig. 2 shows the correlations between the two registered charged fragments. Thus, all the fragments 


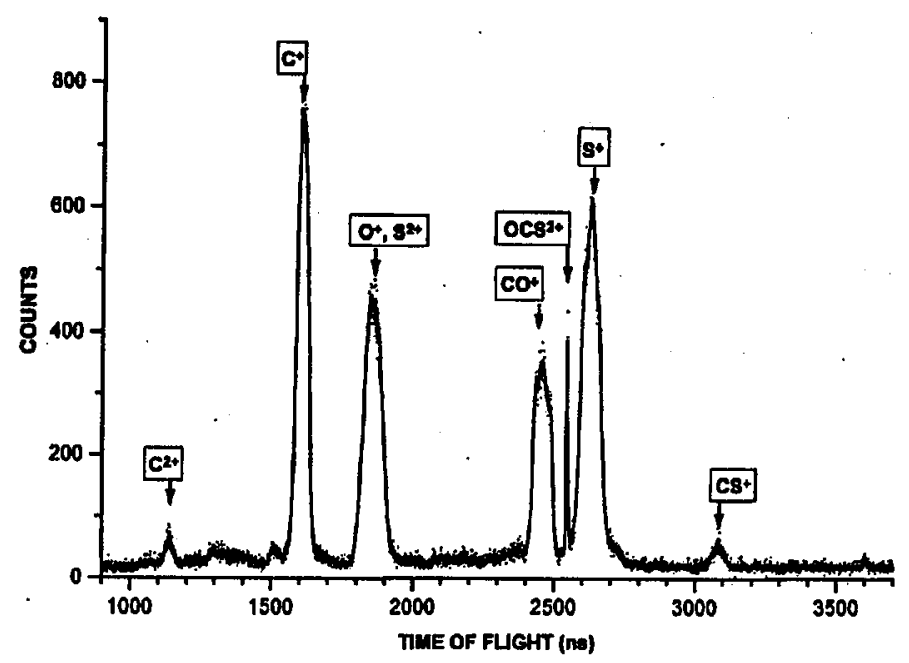

Fig. 1. Photoelectron-photoion coincidence spectrum of fragments of OCS excited near $\mathrm{C}$ 1s edge $(288 \mathrm{eV})$.

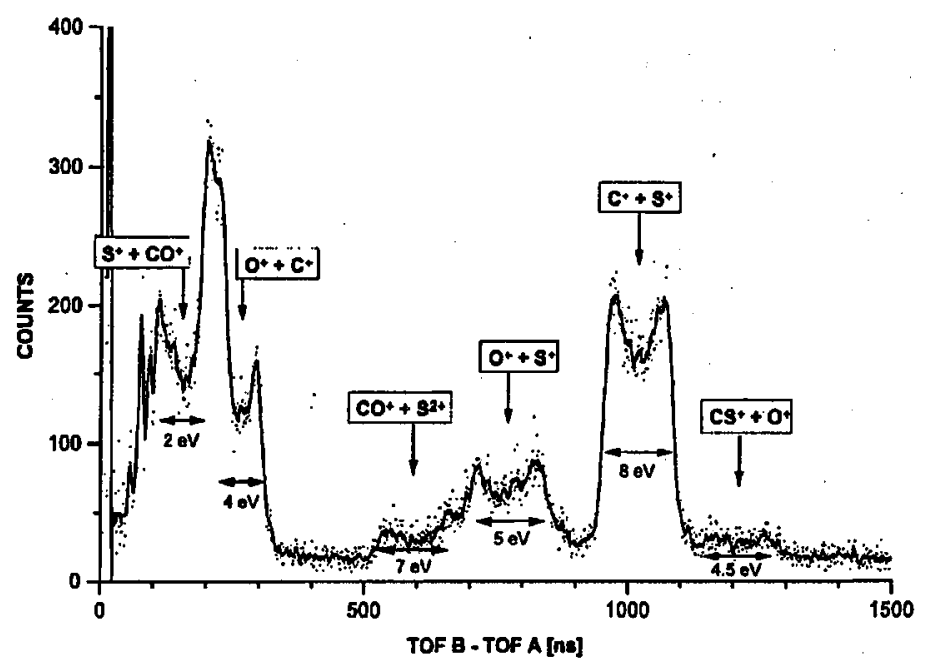

Fig. 2. Photoion-photoion coincidence spectrum of fragments of OCS excited near $\mathrm{C} 1 \mathrm{~s}$ edge $(288 \mathrm{eV})$.

originating from the fragmentations into one charged and one neutral fragment are eliminated from the spectrum. The TOF analysis of the PIPICO spectrum provides information about the energy released during a particular fragmentation process. The measured energies are marked in Fig. 2. However, some of the features in the PIPICO spectrum strongly overlap, making in these cases the analysis difficult if not impossible. 


\subsection{The PEPIPICO experiment}

Performed photoelectron-photoion-photoion coincidence (PEPIPICO) experiment removes these ambiguities. In this type of experiment the correlations between charged fragments are registered in a form of a false colour map shown in Fig. 3.

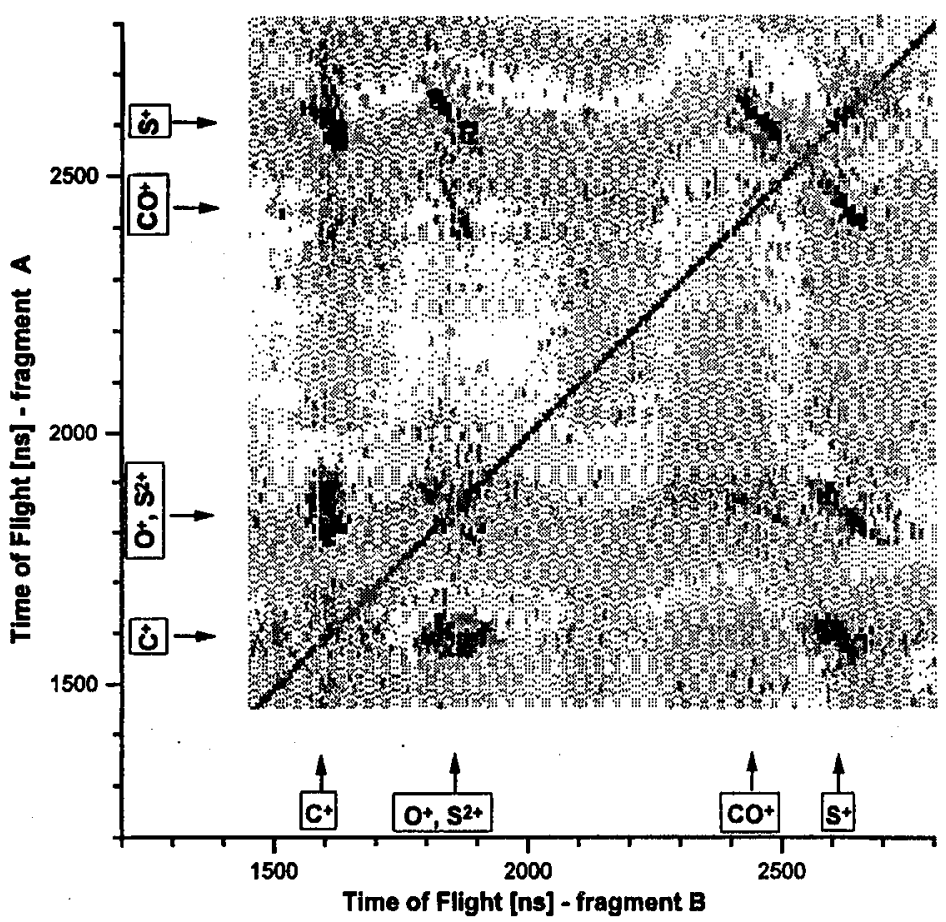

Fig. 3. Photoelectron-photoion-photoion coincidence map of fragmentation of $\mathrm{C} 1 \mathrm{~s}$ excited OCS $(288 \mathrm{eV})$.

The different fragmentation channels are clearly visible in contrast to the PIPICO spectrum. In particular, the $\mathrm{S}^{+}+\mathrm{CO}^{+}$and $\mathrm{O}^{+}+\mathrm{C}^{+}$channels are now well separated and the $\mathrm{O}^{+}+\mathrm{S}^{2+}$ which was entirely buried in the $\mathrm{S}^{+}+\mathrm{CO}^{+}$peak is now evident. Careful analysis of the shapes of the $3 \mathrm{D}$ coincidence features can provide the information about the kinetic energies released and about the possible scenario of the fragmentation processes [2-4].

\section{Conclusions}

The presented data show significant number of possible decay channels of core excited OCS molecule. The striking existence of the stable $\mathrm{OCS}^{2+}$ with the absence of the stable $\mathrm{OCS}^{+}$requires an interpretation. The data are currently being analyzed and more quantitative results will be published. 


\section{References}

[1] P.A. Hatherly, K. Codling, M. Stankiewicz, M. Roper, J. Phys. B 28, 3249 (1995).

[2] J.H.D. Eland, Mol. Phys. 61, 725 (1987).

[3] M. Lavollee, H. Bergeron, J. Phys. B 25, 3101 (1992).

[4] M. Stankiewicz, On Fragmentation of Doubly Ionized Molecules Studied by Triple Coincidence ..., Uniwersytet Jagielloński, Kraków 1994.

[5] W.C. Wiley, I.H. McLaren, Rev. Sci. Instrum. 26, 1150 (1955). 